\title{
Phonological representation of words in working memory during sentence reading
}

\author{
ALBRECHT W. INHOFF, CYNTHIA CONNINE, and BRIANNA EITER \\ State University of New York, Binghamton, New York \\ and \\ RALPH RADACH and DIETER HELLER \\ Technical University, Aachen, Germany
}

\begin{abstract}
The temporal dynamics of a visual target word's phonological representation was examined by presentation of an irrelevant spoken companion word when the participant's eyes reached the target's location during sentence reading. The spoken word was identical, similar, or dissimilar to the phonological specification of the visual target. All spoken words increased the time spent viewing the target, with larger effects in the similar and dissimilar spoken word conditions than in the identical condition. The reading of posttarget text was disrupted when the spoken word was similar but not when it was identical or dissimilar to the target. Phonological interferenceindicates that a word's phonological representation remains active after it has been identified during sentence reading.
\end{abstract}

Learning to read is secondary to the learning of spoken language, and one of the fundamental questions in cognitive psychology and educational research has been whether and how readers use phonological representations for text comprehension. There is now a preponderance of evidence indicating that a visually presented word's phonological representation is determined before its meaning is known (see, e.g., Lee, Rayner, \& Pollatsek, 1999; Perfetti \& Bell, 1991; see also Frost, 1998, for a recent review), although this view is not without its detractors (e.g., Starr \& Fleming, 2001). Moreover, the phonological code of a word appears to remain active after the word has been identified (Folk, 1999; Folk \& Morris, 1995; Inhoff \& Topolski, 1994; McCutchen, Bell, France, \& Perfetti, 1991; McCutchen \& Perfetti, 1982), perhaps because it is used for working memory storage (Baddeley, Logie, Nimmo-Smith, \& Brereton, 1985).

Evidence of the early use of a word's sound code has been obtained in a number of experimental paradigms and tasks, including those involving fluent reading in which available phonological information was manipulated before or immediately after a visual target word was fixated. Using an eye-movement-contingent fast-priming technique, Lee et al. (1999) replaced a visual target word in the sentence with a sequence of uninformative letters until the eyes moved onto the target's location. When this occurred, a word prime was briefly shown at the target location and was followed by the presentation of the target. Measure-

This project was supported by NSF Grant BCS0002024. Correspondence concerning this article should be sent to A. Inhoff, Department of Psychology, State University of New York, Binghamton, NY 139026000 (e-mail: inhoff@binghamton.edu). ment of the ensuing target-viewing duration revealed shorter gaze (defined as the sum of all fixations on the target prior to fixation on another word) for homophonic primes than for graphemically matched controls when prime duration was less than $34 \mathrm{msec}$.

It has also been proposed that a word's phonologicalrepresentation remains active after the word has been recognized. McCutchen et al. (1991) asked readers to judge the meaningfulness of tongue-twister sentences while remembering a set of digits that matched the sound onset properties of the tongue twisters. Control sentences contained a natural mix of onset phonemes. Judgments of meaningfulness took longer and digit recall was more error prone with the tongue twisters, since the similarity of sound onsets between the read and recalled items appeared to hamper their discrimination in phonological working memory.

Readers can also access a word's phonologicalcode after it has been viewed during sentence reading (Folk, 1999; Folk \& Morris, 1995). In Folk and Morris's experiment, readers encountered unambiguous control words and three types of ambiguous targets: heterographic homophones with an ambiguous phonologicalform (e.g., brake/break), homographic homophones with ambiguous orthographic and phonological forms (e.g., calf), and homographic heterophones with an ambiguous orthographic form (e.g., tear). Disambiguating posttarget text was biased toward the less common meaning of the ambiguous form. For instance, in the sentence "There was a tear in a corner of the paper after Jo took it from Tom," the homographic heterophone tear is disambiguated by the subsequent phrase "in a corner of the paper." Measures of word-viewing duration showed that more time was spent reading posttarget words when they referred to the nondominantmeaning of an ambiguous target word than when they followed an unam- 
biguous control word. Critically, rereading occurred for homographic heterophones (e.g., tear) but not for heterographic and homographic homophones (e.g., brake and calf, respectively). According to Folk and Morris (1995; Folk, 1999), heterographic and homographic homophones were not refixated, because their phonological forms were still active in working memory. Homographic heterophones required refixation because their phonological representation in working memory could not be used to retrieve an alternative word meaning.

Prior reading research thus reveals use of phonological representations during word recognition and in working memory during normal sentence reading. It involved, however, comparisons between visual target types, so that effects could depend on specific item properties. Specifically, the phonological forms of ambiguous experimental words could have remained active in working memory because they were likely to assist in the subsequent disambiguation of the targets. In Folk and Morris's (1995) study, there was no attempt to control properties of the disambiguating information, which could have been obtained either immediately after the reading of the ambiguous target word or after the reading of several intervening words. The present study had two goals: first, to determine whether the phonological form of a visual word remains active after its recognition when the word has no distinguishing phonological properties and, second, to determine the time course of phonological form activation during normal reading.

This was accomplished by using on-line recordings of eye movements to manipulate the phonological environment within which a visual target word was read. Specifically, a visual target word and a spoken companion word were presented together when the eyes moved to the right of a predefined sentence location. The relationship between the visual target and its spoken mate was manipulated so that their phonological forms were identical, similar, or dissimilar. The phonological environment within which a visual target was read was thus manipulated while the visual target word remained constant. None of the targets was phonologically ambiguous, and all were embedded in relatively simple declarative sentences. The time that the participants spent viewing the target and subsequent words in the sentence was measured and used to determine whether and how sound similarity influenced target and posttarget reading.

We hypothesized that high phonological similarity would hamper ongoing processing of the visual target, because text- and speech-based sound codes should be difficult to discriminate. Consequently, readers should spend more time gazing at the visual target in the similar condition than in the identical or dissimilar condition. Critically, if the sound codes of the visual target and of the spoken companion word remained active after they were identified, then their interference in working memory should also increase the time spent on reading posttarget words. To determine whether the duration of a spoken word influenced the time course of its effect on target and posttarget reading, we created two speech tokens: one with a standard articulation duration and one with a temporally shortened duration.

\section{METHOD}

\section{Participants}

Sixty-six undergraduate and graduate students with no history of reading disabilities participated in the study for course credit or payment.

\section{Materials}

The reading material consisted of 56 declarative sentences (42 experimental sentences and 14 fillers). Each experimental sentence contained a nonhomophonic visual target word (a noun) that occurred within the first half of the sentence. The mean CELEX word frequency (Baayen, Piepenbrock, \& van Rijn, 1993) of the targets was 17 per million, with a range from .5 to 129 per million. Thirty targets were monosyllabic, and the remaining 12 were bisyllabic.

Three to-be-spoken words (nouns or adjectives) were selected for each visual target: one that was identical in phonological form and meaning to the visual target, one that was similar in phonological form and dissimilar in meaning, and one that was dissimilar in both phonological form and meaning. For instance, the visual target word plate was presented with the spoken word "plate" in the identical condition, with "place" in the similar condition, and with "horse" in the dissimilar condition. The phonological form of a similar spoken word overlapped with the phonological form of the target except for one or two noninitial phonemes. Furthermore, the orthographic forms of the similar word and the target were neighbors in that they differed by one letter. Identical and dissimilar spoken words were matched for syllable number but did not share any phonemes or letters at corresponding word locations.

A rating task was conducted to examine how well similar and dissimilar spoken words fit into prior sentence context to exclude the possibility that one of the two spoken word types would offer a better contextual fit. Sentence fragments up to the target word were presented to 32 participants who had not participated in the reading experiment. Half of the sentence fragments were followed by a written version of the similar spoken word, and the other half were followed by a written version of the corresponding dissimilar word. Two lists were constructed; sentence fragments followed by a similar word on one list were followed by a dissimilar word on the other. The rating scale ranged from 1 (poor fit) to 7 (excellent fit). To prevent a bias toward low ratings, the materials also included fragments of filler sentences that were always followed by the context-consistent visual filler word. The results showed mean ratings of 2.0 for similar words and 1.9 for dissimilar words $(t=0.6, p>.5)$; filler fragments received a rating of 6.2. Thus, both similar and dissimilar words were equally poor semantic fits to their companion visual sentences.

All spoken words were articulated with normal tempo by a native speaker of American English and digitally recorded at $22 \mathrm{KHz}$ and at 16-bit resolution. The durations of the words' naturally produced articulations ranged from approximately $350 \mathrm{msec}$ to $700 \mathrm{msec}$, with a mean of $546 \mathrm{msec}$ for identical spoken words and of $534 \mathrm{msec}$ for similar and dissimilar spoken words. A speech compression routine (Sound Forge 4.5) was used to shorten the duration of each speech token by one third. Two native speakers verified that these shortened speech tokens could be readily identified. Thirty readers listened to standard-speech tokens presented via loudspeakers, and 36 listened to shortened tokens presented via headphones. No spoken word was presented during the reading of filler sentences. Instead, a burst of white noise was presented upon the fixation of a filler target to assess the oculomotor system's response to the onset of an auditory signal. Two durations were used: one that corresponded to the mean of the standard spoken words and one that corresponded to the mean of the short-duration words. 


\section{Apparatus}

Text was displayed in black on a gray background at a resolution of $1,028 \times 720$ pixels. Black Courier type font was used, and the distance between each reader's eyes and the monitor was set to approximately $85 \mathrm{~cm}$. At this distance, each letter subtended $0.44^{\circ}$ of visual angle laterally.

Eye movements and head position were recorded with an EyeLink tracking system at a rate of $250 \mathrm{~Hz}$ and with a relative visual resolution of approximately $0.25^{\circ}$. On-line specifications of viewing location were used to implement eye-movement-contingent stimulus presentations and to compute the different measures of oculomotor activity during sentence reading. Acoustic signal presentation was controlled by SoundblasterPro hardware. All acoustic signals were presented at a comfortable listening level of approximately $70 \mathrm{~dB}$.

\section{Procedure}

The experimental session began with a calibration that was considered successful when the fixations of left-side, central, and rightside fixation markers were within $1 / 4^{\circ}$ of the respective marker. Thereafter, the participants read a sequence of unrelated sentences for comprehension while their eye movements were monitored. One sentence was shown at a time, and the reader controlled the onset and offset of the sentence by pressing the space bar on a keyboard. The words of a sentence were continuously visible during sentence reading, except for the visual target of an experimental sentence and the filler word of a filler sentence. A placeholder, consisting of a pseudoword, occupied the corresponding location at the onset of sentence reading. The phonological form of the placeholder was unrelated to the phonological form of the corresponding visual target and to the phonological forms of the target's three spoken companion words. The placeholder was replaced with the target, and the spoken companion word was presented when the eyes moved to the right of the blank space preceding the target location (i.e., the target boundary).
Implementation of the visual display change took 13 to $18 \mathrm{msec}$. However, the eye was in motion when the display change routines were initiated, and the target was generally visible at the onset of a fixation or less than $10 \mathrm{msec}$ thereafter. Speech onset occurred within $10 \mathrm{msec}$ of boundary crossing.

A sample trial is shown in Figure 1.

Readers were told that either a word or noise would be presented at some point in time during sentence reading. They were explicitly told that their knowledge of the spoken word would not be tested and that they should focus on sentence meaning instead. They were asked to paraphrase the previously read sentence approximately every 10th trial, and they did this successfully ( $>98 \%$ correct). They never reported the spoken word in lieu of the visual target.

\section{Design}

Three lists were used, so that each visual target was paired with each spoken word type and each list contained the same number of sentences in each one of the experimental conditions. The spoken words used in the similar condition of one list were paired with a different-sounding visual target on another list to create the dissimilar condition.

Following other studies of sentence reading (e.g., Lee et al., 1999), we used gaze duration as the primary index of a word's processing. Five consecutive words were analyzed: the pretarget $(N-1)$, the target $(N)$, and the three following words $(N+1$ to $N+3)$. An omnibus analysis of variance was used to determine effects of the spoken word's articulation duration (normal vs. shortened), spoken word type (identical vs. similar vs. dissimilar), and position $(N-1$ to $N+3)$. This was followed by separate examinations of spoken word type effects.

Readers were allowed to recheck the visual target-for example, when the similar spoken word was mistaken for the phonological form of the visual target, or, if the flow of activation between orthography and phonology was bidirectional (see, e.g., Stone, Van-

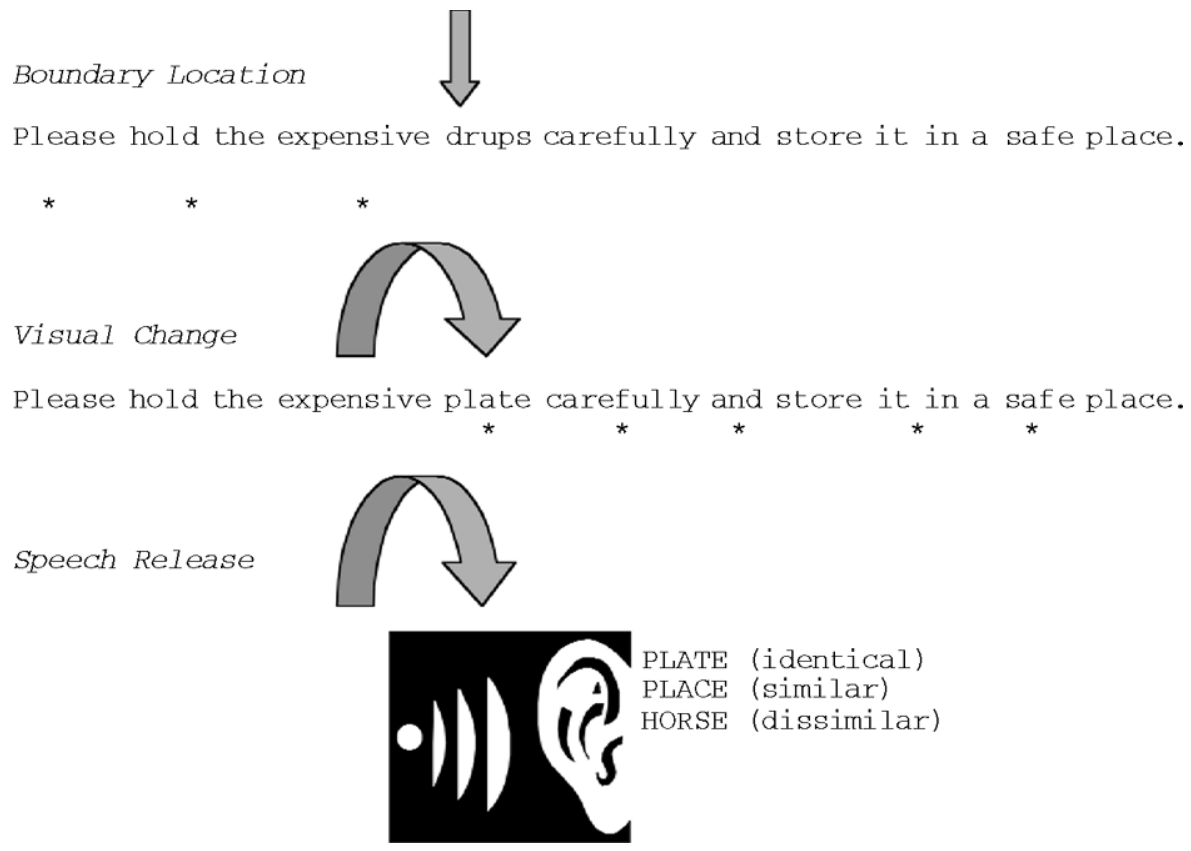

Figure 1. A sample sentence along with a progression of fixations, indicated by asterisks below the line of text. The boundary location and the occurrence of audiovisual changes is marked by arrows. Movement of the eyes to the right of the boundary resulted in the unmasking of the target word, which was masked until then. The same eye movement also initiated the spoken word presentation. Only one spoken word was triggered at each trial. 
hoy, \& van Orden, 1997), when the graphemic form of the similar spoken word was mistaken for the graphemic form of the target. To capture spoken word effects on rechecking, we computed a supplementary target-viewing measure, total viewing duration, which consisted of the target's gaze duration and the time spent rereading it.

In two other supplementary analyses, we examined whether posttarget effects were due to specific listening strategies or to instances in which the eyes left the target before the spoken word was fully articulated. Specifically, we examined spoken word effects for the first three trials of each condition, the assumption being that a strategy was not established at the very onset of the experiment. We also used a median split of target gaze durations to form two groups of readers: one with relatively short and one with relatively long target gazes. This was done to determine whether spoken word effects on posttarget reading occurred primarily when readers had spent relatively little time viewing the target. More details about the setup, procedure, and other aspects of the data are described in Inhoff, Connine, and Radach (2002).

\section{RESULTS}

Spoken word duration did not influence sentence reading in the omnibus analysis. Mean gaze durations in the standard- and short-duration conditions were $362 \mathrm{msec}$ and $373 \mathrm{msec}$, respectively $\left[F_{1}\right.$ and $\left.F_{2}=2.40, p>.12\right]$. Furthermore, speech duration did not interact with any other effect (all $\left.F_{\mathrm{S}}<1\right)$.

Figure 2 shows the gaze durations for the five critical words of a sentence as a function of spoken word type, averaged over spoken word duration.
All three spoken word types disrupted target reading. Target gaze durations were longer than any other gaze duration, resulting in a highly reliable effect of position $\left[F_{1}(4,256)=60.4, F_{2}(4,328)=76.8\right.$, both $p$ s $\left.<.01\right]$. White noise, presented during the reading of filler sentences, also increased gaze durations for the concurrently viewed word. Specifically, gazes on $N-1$ to $N+3$ words of filler sentences were 258,450,338, 342, and $380 \mathrm{msec}$, respectively.

Critically, gaze durations during the reading of experimental sentences were influenced by the phonological properties of the spoken word. Figure 2 reveals a large main effect of word type $\left[F_{1}(2,128)=10.0, F_{2}(2,164)=\right.$ 7.6 , both $p \mathrm{~s}<.01]$ and a different time course of similar and dissimilar spoken word effects. This was expressed in an interaction of word position and spoken word type $\left[F_{1}(8,512)=5.0, F_{2}(8,656)=3.6\right.$, both $p$ s $\left.<.01\right]$. Effects of a dissimilar spoken word were short-lived and confined to the reading of the visual target. Phonologically similar effects, in contrast, were longer lasting and extended into the reading of posttarget words. This was confirmed by additional comparisons of spoken word effects for the target word and the next three words in the text.

For the target word, gazes in the identical condition were shorter $(24 \mathrm{msec})$ than gazes in the similar condition $\left[F_{1}(1,64)=5.4, p<.05 ; F_{2}(1,83)=5.0, p<.05\right]$ and shorter $(25 \mathrm{msec})$ than gazes in the dissimilar condition $\left[F_{1}(1,64)=10.4, p<.01 ; F_{2}(1,83)=3.1, p<.08\right]$. The

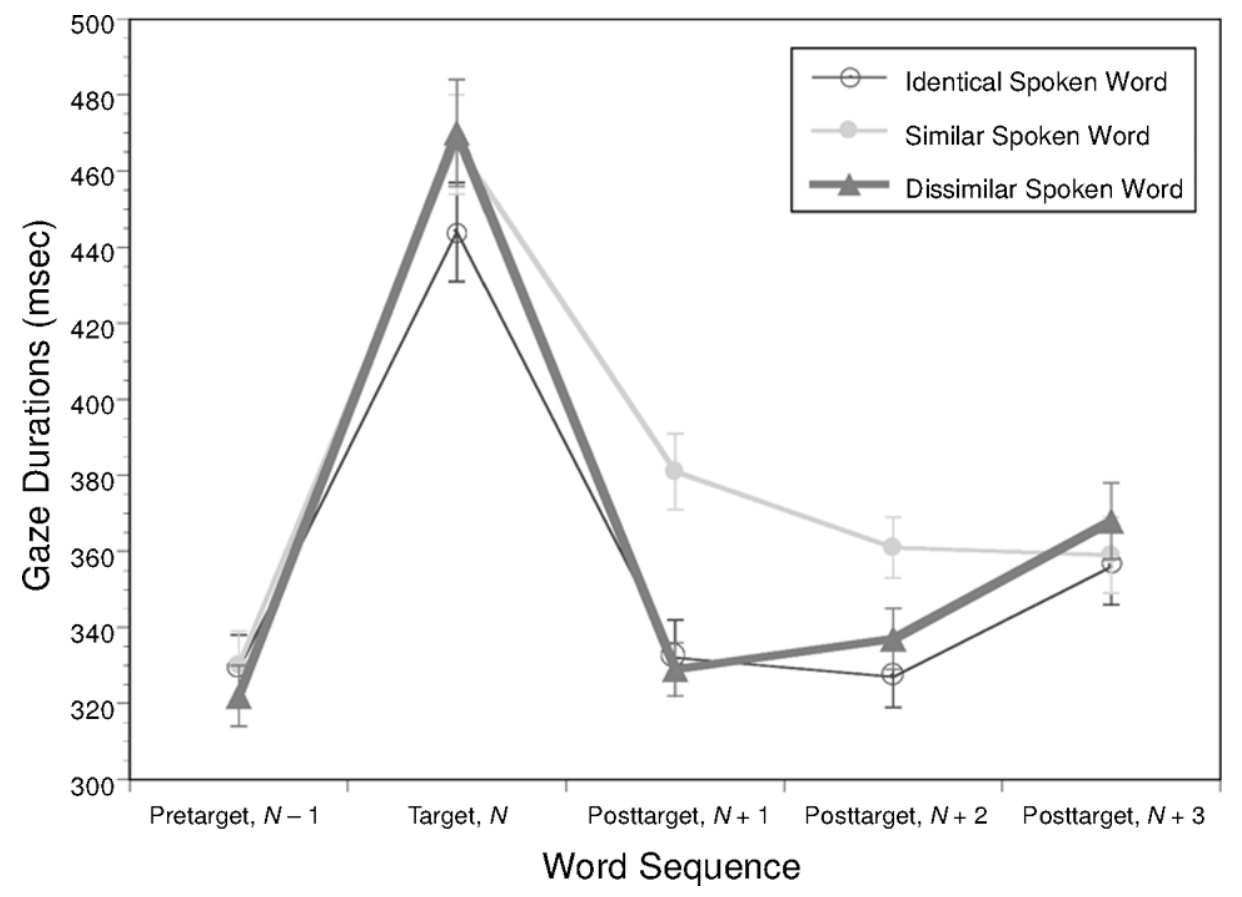

Figure 2. Gaze durations on the five critical words of a sentence, beginning with the pretarget word $\mathrm{N-1}$, as a function of spoken word type. The effects are collapsed across the two spoken word rates. 
similar and dissimilar conditions did not differ $\left(F_{1}\right.$ and $\left.F_{2}<1\right)$. The effect pattern changed for $N+1$, with longer gazes in the similar condition than in either the dissimilar condition $\left[F_{1}(1,64)=38.3, F_{2}(1,82)=23.9\right.$, both $p \mathrm{~s}<$ $01]$ or the identical condition $\left[F_{1}(1,64)=26.1, F_{2}(1,82)=\right.$ 37.2 , both $p$ s $<.01]$. Identical and dissimilar spoken word conditions did not differ $\left[F_{1}\right.$ and $\left.F_{2}<1\right]$. Gaze duration for $N+2$ also revealed selective interference, again with a longer duration in the similar condition than in either the dissimilar condition $\left[F_{1}(1,64)=9.5, F_{2}(1,82)=10.9\right.$, both $p \mathrm{~s}<.01]$ or the identical condition $\left[F_{1}(1,64)=5.70\right.$, $\left.p<.025 ; F_{2}(1,82)=11.3, p<.01\right]$. As before, the difference between the identical and dissimilar conditions was negligible [ $F_{1}$ and $\left.F_{2}<1\right]$. Gazes on word $N+3$, as the end of the sentence was approached, were longer than gazes on words $N+1$ and $N+2$ in all three experimental conditions (and filler trials). None of the spoken word type effects was significant (all $F_{\mathrm{S}}<1$ ).

Together, the pattern of gaze durations revealed immediate effects of the spoken word type on target viewing. More importantly, it also revealed longer gazes for words $N+1$ and $N+2$ when the spoken companion word and the previously read visual target were phonologically similar then when they were dissimilar.

\section{Supplementary measures}

Total viewing durations in the identical, similar, and dissimilar conditions were 516, 600, and $589 \mathrm{msec}$, respectively. Critically, the similar and dissimilar conditions thus differed by a mere $11 \mathrm{msec}\left(F_{1}\right.$ and $\left.F_{2}<1\right)$. If readers had checked the visual target to determine whether it was read correctly in the similar spoken word condition, then total viewing durations should have been selectively increased in that condition. Examinations of spoken word effects for the first three trials of each condition, presented in Table 1 , show deleterious effects of similarity for $N+1$ and $N+2$, which argues against the possibility that spoken word effects were due to listening strategies (no inferential statistics were computed owing to the small number of trials in each condition). Finally, examination of posttarget gazes as a function of readers' target-viewing duration, presented in Table 2, shows similarity interference for the group of readers with short (380-msec) and long (546-msec) target gazes. The three-way interaction of target gaze, spoken word type, and posttarget location $(N+1$
Table 1

Posttarget Gaze Durations (in Milliseconds) for the First Three Trials in Each of the Three Spoken Word Conditions

\begin{tabular}{cccc}
\hline & \multicolumn{3}{c}{ Spoken Companion Word } \\
\cline { 2 - 4 } Posttarget & Identical & Similar & Dissimilar \\
\hline$N+1$ & 354 & 400 & 346 \\
$N+2$ & 330 & 379 & 358 \\
$N+3$ & 382 & 392 & 398 \\
\hline
\end{tabular}

to $N+3)$ was negligible $(F<1)$. A complementary analysis, in which we examined target gaze durations as a function of spoken word duration, was also negative. Across the 168 spoken words, there were 125 different spoken word durations, and the correlation between spoken word durations and the mean target gaze durations was also negligible $[r(124)=-.11]$.

\section{DISCUSSION}

The main goal of the present study was to determine whether the sound code of an unambiguous visual target word remained active after it was viewed during sentence reading. In support of this view, a spoken companion word presented during the viewing of a visual target resulted in longer posttarget viewing durations when its phonological code was similar to that of the target than when it was either identical or dissimilar to that of the target. Supplementary analyses revealed that sound similarity hampered posttarget reading from the very onset of the experiment, indicating that posttarget effects were not the result of a particular listening strategy and that deleterious effects of sound similarity on posttarget reading were not confined to instances in which the eyes moved off the visual target word before the spoken companion word was fully articulated. Together, these results demonstrate that a word's phonological representation remains active, as it did in the research of Folk (1999; Folk \& Morris, 1995), even when the representation is not likely to assist subsequent lexical disambiguation. Continued representation of an identified word's phonologicalcode thus appears to be the rule rather than the exception.

Analyses of the visual target showed that it was not mistaken for the similar spoken companion word, since sound similarity did not lead to rereading. Furthermore, on the

Table 2

Posttarget Gaze Durations (in Milliseconds) for Readers With Relatively Short and Long Prior Target Gazes, With Standard Errors of the Means

\begin{tabular}{|c|c|c|c|c|c|c|c|c|c|c|c|c|}
\hline \multirow{4}{*}{$\begin{array}{l}\text { Posttarget } \\
\text { Word }\end{array}$} & \multicolumn{12}{|c|}{ Spoken Companion Word } \\
\hline & \multicolumn{4}{|c|}{ Identical } & \multicolumn{4}{|c|}{ Similar } & \multicolumn{4}{|c|}{ Dissimilar } \\
\hline & \multicolumn{2}{|c|}{$\begin{array}{c}\text { Short } N \\
\text { Target Gaze } \\
\end{array}$} & \multicolumn{2}{|c|}{$\begin{array}{c}\text { Long } N \\
\text { Target Gaze } \\
\end{array}$} & \multicolumn{2}{|c|}{$\begin{array}{c}\text { Short } N \\
\text { Target Gaze } \\
\end{array}$} & \multicolumn{2}{|c|}{$\begin{array}{c}\text { Long } N \\
\text { Target Gaze } \\
\end{array}$} & \multicolumn{2}{|c|}{$\begin{array}{c}\text { Short } N \\
\text { Target Gaze } \\
\end{array}$} & \multicolumn{2}{|c|}{$\begin{array}{c}\text { Long } N \\
\text { Target Gaze } \\
\end{array}$} \\
\hline & $M$ & $\overline{S E}$ & $M$ & $\overline{S E}$ & $M$ & $\overline{S E}$ & $M$ & $\overline{S E}$ & $M$ & $\overline{S E}$ & $M$ & $\overline{S E}$ \\
\hline$N+1$ & 312 & 14 & 352 & 14 & 355 & 15 & 406 & 15 & 306 & 10 & 351 & $\overline{10}$ \\
\hline$N+2$ & 312 & 12 & 343 & 12 & 345 & 13 & 378 & 13 & 312 & 11 & 362 & 11 \\
\hline$N+3$ & 336 & 15 & 376 & 15 & 349 & 14 & 369 & 14 & 336 & 15 & 376 & 15 \\
\hline
\end{tabular}


approximately 180 trials in which memory for sentence content was probed, the readers never reported the similar spoken word in lieu of the visual target, and similar and dissimilar spoken words were equally disruptive to target reading relative to the identical condition. ${ }^{1}$ We take this to mean that the similar spoken companion word did not influence the prelexical phonological processing of the visual target, perhaps because this phonological code was determined relatively quickly_within less than $40 \mathrm{msec}$ of fixation onset (Lee et al., 1999; Perfetti \& Bell, 1991) and/or because the phonological code used during the initial stage of visual word recognition differs in fundamental ways from the phonological code used for spoken word identification.

As was noted before, a similar spoken word selectively interfered with posttarget reading, suggesting that the phonological code available during posttarget reading differs from that available while the target is fixated. Visual and spoken words could activate structurally equivalent working memory representations, so that they are difficult to discriminate during posttarget reading (McCutchen et al., 1991).

The strength of the working memory code is subject to change. Deleterious effects of phonological similarity, expressed in the difference between similar and dissimilar conditions, decreased from $53 \mathrm{msec}$ during $N+1$ reading to $24 \mathrm{msec}$ during $N+2$ reading, and they ceased to exist during $N+3$ reading. A word's working memory representation may thus either remain active until sentence integration is completed (Folk, 1999; Folk \& Morris, 1995) or be subject to relatively rapid loss of strength, irrespective of the success of ongoing sentence comprehension. We are currently examining the two hypotheses.

\section{REFERENCES}

BAayen, R. H., Pie Penbrock, R., \& van Rijn, H. (1993). The CELEX lexical database (CD-ROM). Philadelphia: Linguistic Data Consortium, University of Pennsylvania.

Baddeley, A. D., Logie, R. H., Nimmo-Smith, I., \& Brereton, I. J. (1985). Components of fluent reading. Journal of Memory \& Language, 24, 119-131.
FOLK, J. R. (1999). Phonological codes are used to access the lexicon during silent reading. Journal of Experimental Psychology: Learning, Memory, \& Cognition, 25, 892-906.

FOLK, J. R., \& MORRIS, R. K. (1995). Multiple lexical codes in reading: Evidence from eye movements, naming time, and oral reading. Journal of Experimental Psychology: Learning, Memory, \& Cognition, 21, 1412-1429.

FROST, R. (1998). Toward a strong phonological theory of visual word recognition. Psychological Bulletin, 123, 71-99.

InhoFf, A. W., Connine, C., \& RADACH, R. (2002). A contingent speech technique in eye movement research on reading. Behavior Research Methods, Instruments, \& Computers, 34, 471-480.

INHOFF, A. W., \& TOPOLSKI, R. (1994). Use of phonological codes during eye fixations in reading and in on-line and delayed naming tasks. Journal of Memory \& Language, 33, 689-713.

Lee, H.-W., Rayner, K., \& Pollatse K, A. (1999). The time course of phonological, semantic, and orthog raphic coding in reading: Evidence from the fast-priming technique. Psychonomic Bulletin \& Review, 6 , 624-634.

McCutchen, D., Bell, L. C., France, I. M., \& Perfetti, C. A. (1991). Phoneme specific interference in reading: The tongue-twister effect revisited. Reading Research Quarterly, 26, 87-103.

McCutchen, D., \& Perfetti, C. A. (1982). The visual tongue-twister effect: Phonological activation in silent reading. Journal of Verbal Learning \& Verbal Behavior, 21, 672-687.

Perfetti, C. A., \& Bell, L. (1991). Phonemic activation during the first $40 \mathrm{~ms}$ of word identification: Evidence from backward masking. Journal of Memory \& Language, 30, 473-485.

StARr, M. S., \& Fleming, K. (2001). A rose by another name is not the same: The role of orthographic knowledge in homophone confusion errors. Journal of Experimental Psychology: Learning, Memory, \& Cognition, 27, 744-760.

Stone, G. O., Vanhoy, M., \& Van Orden, G. C. (1997). Perception is a two-way street: Feedforward and feedback phonologyin visual word recognition. Journal of Memory \& Language, 36, 337-359.

\section{NOTE}

1. The source of the identity effect during target viewing, defined as shorter target gazes in the identical condition than in either the similar or the dissimilar condition, remains unclear, since the phonological forms and the meanings of similar and dissimilar companion words differed from those of the visual target.

(Manuscript received June 17, 2002; revision accepted for publication January 29, 2003.) 